\title{
On the dichotomy of Perron numbers and
}

\author{
beta-conjugates
}

\section{Jean-Louis Verger-Gaugry *}

\begin{abstract}
Let $\beta>1$ be an algebraic number. A general definition of a beta-conjugate of $\beta$ is proposed with respect to the analytical function $f_{\beta}(z)=-1+\sum_{i \geq 1} t_{i} z^{i}$ associated with the Rényi $\beta$-expansion $d_{\beta}(1)=0 . t_{1} t_{2} \ldots$ of unity. From Szegö's Theorem, we study the dichotomy problem for $f_{\beta}(z)$, in particular for $\beta$ a Perron number: whether it is a rational fraction or admits the unit circle as natural boundary. The first case of dichotomy meets Boyd's works. We introduce the study of the geometry of the beta-conjugates with respect to that of the Galois conjugates by means of the Erdős-Turán approach and take examples of Pisot, Salem and Perron numbers which are Parry numbers to illustrate it. We discuss the possible existence of an infinite number of beta-conjugates and conjecture that all real algebraic numbers $>1$, in particular Perron numbers, are in $\mathrm{C}_{1} \cup \mathrm{C}_{2} \cup \mathrm{C}_{3}$ after the classification of Blanchard/Bertrand-Mathis.
\end{abstract}

2000 Mathematical Subject Classification: 11M99, 03D45, 30B10, 12 Y05.

*Work supported by ACINIM 2004-154 "Numeration" 
Key words: Perron number, Pisot number, Salem number, Erdős-Turán's Theorem, numeration, Szegö's Theorem, uniform distribution, beta-shift, zeroes, betaconjugate. 


\section{Contents}

1 Introduction . . . . . . . . . . . . . . . . . 3

2 Locus of zeroes . . . . . . . . . . . . . . . . 8

3 Beta-conjugates, Galois conjugates and dichotomy . . . . . . . . . . . . 14

3.1 The rational fraction case . . . . . . . . . . . . . . 15

3.2 The second case: Hadamard type theorems, frequencies of digits . . . 17

4 Erdös-Turán approach . . . . . . . . . . . . . . . . . 21

4.1 Uniform clustering near the unit circle . . . . . . . . . . . . . 21

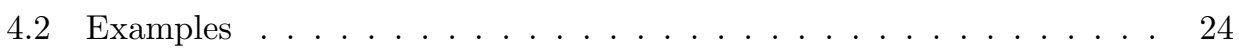

4.2.1 Selmer's Perron numbers in the class $C_{1} \ldots \ldots . \ldots 24$

4.2.2 Bassino's infinite family of cubic Pisot numbers . . . . . . . . 26

4.2.3 Boyd's infinite families of Pisot and Salem numbers . . . . . . 28

4.2.4 Confluent Parry numbers . . . . . . . . . . . . . . . . 32

\section{Introduction}

This note is concerned with the rich interplay between number theory and dynamical systems of numeration, for which numeration means numeration in base $\beta$, where $\beta$ is a real algebraic number $>1[2][7][8][31][38]$.

Let us recall some definitions to fix notations. The real number $\beta>1$ is a Perron number if and only if it is an algebraic integer and all its Galois conjugates $\beta^{(i)}$ satisfy: 
$\left|\beta^{(i)}\right|<\beta$ for all $i=1,2, \ldots, d-1$, if $\beta$ is of degree $d \geq 1\left(\right.$ with $\beta^{(0)}=\beta$ ). Let $\mathbb{P}$ be the set of Perron numbers. We will assume throughout the paper that the numeration basis $\beta$ is noninteger. The Rényi $\beta$-expansion of 1 is by definition denoted by

$$
d_{\beta}(1)=0 . t_{1} t_{2} t_{3} \ldots \quad \text { and corresponds to } \quad 1=\sum_{i=1}^{+\infty} t_{i} \beta^{-i}
$$

where $t_{1}=\lfloor\beta\rfloor, t_{2}=\lfloor\beta\{\beta\}\rfloor, t_{3}=\lfloor\beta\{\beta\{\beta\}\}\rfloor, \ldots(\lfloor x\rfloor$ and $\{x\}$ denote the integer part, resp. the fractional part, of a real number $x$ ). The digits $t_{i}$ belong to the finite alphabet $\mathcal{A}_{\beta}=\{0,1,2, \ldots,\lfloor\beta\rfloor\}$ by construction. We will say that $\beta$ is a Parry number (previously called a beta-number by Parry [36]) if $d_{\beta}(1)$ is finite or ultimately periodic (i.e. eventually periodic); in particular, we say that a Parry number $\beta$ is simple if $d_{\beta}(1)$ is finite.

Parry [36] has shown that Parry numbers are algebraic integers. Parry numbers (simple or non-simple) are Perron numbers (Theorem 7.2.13 and Proposition 7.2.21 in Lothaire [31]). The converse is wrong, somehow mysterious. This defines a dichotomy in $\mathbb{P}$ : the subset of Perron numbers which are Parry numbers, the subset of $\mathbb{P}$ which are not Parry numbers.

The main motivation of the present work is the following: on one hand, $d_{\beta}(1)=$ $0 . t_{1} t_{2} \ldots$ entirely controls the $\beta$-shift [11] making the sequence $\left(t_{i}\right)_{i \geq 1}$ a very important one with values in the alphabet $\mathcal{A}_{\beta}$, on the other hand in the philosophy of Pólya and Ostrowski, an analytic function is entirely defined by the coefficient vector of its Taylor series at one point of its domain of definition. Here, the idea consists in studying the $\beta$-shift by means of the Taylor series $f_{\beta}(z)$ defined below in (1.2), where 
precisely its coefficient vector is exactly the sequence $\left(t_{i}\right)_{i \geq 0}$ up to the constant term -1 . Then the correspondance between the Rényi $\beta$-expansion $d_{\beta}(1)$ of unity and the analytical function $f_{\beta}(z)$ becomes canonical, what allows the exploration of the $\beta$-shift in terms of analytical functions. What appears first is the possible existence of beta-conjugates, whose exploration was made first by Boyd [14] for Pisot numbers (Section 4).

We explore in particular in this note the set of Perron numbers, and their betaconjugates, by means of the Erdős-Turán approach [10] [20] [26] [28] [34] applied to the analytical function $f_{\beta}(z)$ or to its family of polynomial sections, using Hurwitz Theorem ([33] p. 4).

Let us give the definition of $f_{\beta}(z)$ and the only two forms that can be taken by this function. Let $\beta>1$ be a real number and $d_{\beta}(1)=0 . t_{1} t_{2} \ldots$. Let $t_{0}=-1$. Define

$$
f_{\beta}(z):=\sum_{i=0}^{+\infty} t_{i} z^{i}
$$

of the complex variable $z$. If $d_{\beta}(1)$ is infinite, its radius of convergence is 1 .

Dichotomy principle (D): by Szegö's Theorem [46], [19] p 324-7, addressed to power series with coefficients in a finite subset of $\mathbb{C}$ (the alphabet $\mathcal{A}_{\beta}$ ), we deduce (Section 3 ) that $f_{\beta}(z)$ is either equal to

(i) $U(z)+z^{m+1} \frac{V(z)}{1-z^{n+1}}$ where $U(z)=-1+\sum_{i=1}^{m} a_{i} z^{i}, V(z)=\sum_{i=0}^{n} b_{i} z^{i}$ are polynomials with coefficients in $\mathcal{A}_{\beta}$ and $m \geq 1, n \geq 0$ rational integers, for which the respective strings of coefficients $\left(a_{i}\right)_{i},\left(b_{i}\right)_{i}$ satisfy the admissibility conditions of Parry [36] [23] [24], or 
(ii) $f_{\beta}(z)$ is an analytic function which is not continued beyond the unit circle (which is its natural boundary).

Szegö's theorem is a generalization of simpler theorems due to Borel, Carlson, Fatou, Pólya [18] [37]. The dichotomy of $\mathbb{P}$ corresponds exactly to this dichotomy of analytical functions, the first case corresponding exactly to the definition of a Parry number; in particular the case of a simple Parry number corresponds to $V(z) \equiv 0$.

The power series $f_{\beta}(z)$ is related to the zeta function of beta-transformation, i.e. $\hat{\zeta_{\beta}}(z)$ in the sense of Takahashi [48], or $\zeta_{\beta}(z)$ in the sense of Flatto, Lagarias and Poonen [21] (Appendix in [21] for comparison) :

$$
f_{\beta}(z)=\frac{-1}{\hat{\zeta_{\beta}(z)}}=\frac{-1}{\zeta_{\beta}(z)} \quad \text { for } \beta \text { a non-simple Parry number; }
$$

see also Solomyak [45], Boyd [14]. The advantage of working with $f_{\beta}(z)$ is that the coefficients $t_{i}$ lie in a finite subset of $\mathbb{C}$, what allows the simultaneous application of the dichotomy principle (D) and of the Erdős-Turán approach, while it is not the case for the coefficients of the Taylor series (at $z=0$ ) of $\zeta_{\beta}(z)$, resp. $\hat{\zeta_{\beta}}(z)$. In this correspondance (1.3) poles become zeroes. We will take the following definition for a beta-conjugate; this definition coincides with that of Boyd [14] in the case where $\beta$ is a non-simple Parry number.

Definition 1.1. Let $\beta>1$ be an algebraic number. A beta-conjugate of $\beta$ is the inverse $\xi^{-1}$ of a complex number $\xi$ such that

$$
f_{\beta}(\xi)=0, \quad \text { with } \xi^{-1} \neq \text { any Galois conjugate } \beta^{(i)} \text { of } \beta .
$$


The set of zeroes of $f_{\beta}(z)$ is the disjoint union of a subset of inverses of Galois conjugates of $\beta$ and the subset of the inverses of the beta-conjugates of $\beta$. In the case (D)(i) the subset of Galois conjugates concerned contains all the Galois conjugates. In the case (D)(ii) this subset may contain (see (P4) below, and Section 3.1) no Galois conjugate of $\beta$ of modulus strictly greater than 1 .

Since the existence of beta-conjugates is still mysterious, the following problems are relevant (for $\beta>1$ an algebraic number):

Case $(\mathbf{D})(\mathrm{i})$ :

(P1) What is the number and the geometry of the beta-conjugates of $\beta$ with respect to the geometry of the Galois conjugates of $\beta$, as a function of the sequence of digits $\left(t_{i}\right)$ in $d_{\beta}(1)$ ?

Case (D)(ii):

(P2) Under which condition(s) is the number of beta-conjugates of $\beta$ empty, finite, or infinite?

(P3) Are the beta-conjugates of $\beta$ algebraic or transcendental numbers $\left(f_{\beta}(z)\right.$ being transcendental)?

(P4) Does the analytical function $f_{\beta}(z)$ (knowing that $f_{\beta}\left(\beta^{-1}\right)=0$ ) cancel at all the inverses $\beta^{(i)^{-1}}$ of the Galois conjugates $\beta^{(i)}$ of $\beta$ which are such that $\left|\beta^{(i)}\right|>1$ ?

Concerning the set of Perron numbers $\mathbb{P}$ and its arithmetics (Lind [30] pp 292-5):

(P5) How does the set of Perron numbers spread over the two cases of (D)? 
(P6) What are the relative proportions of irreducible Perron numbers in (D)(i) and in $(\mathbf{D})(\mathrm{ii})$ ?

Recall that the set of simple Parry numbers (Theorem 5 in [36]), resp. the set $\mathbb{P}$ of Perron numbers (Proposition 2 in $[30])$, is dense in $(1,+\infty)$. The (non-unique) factorization of a Perron number $\beta=\beta_{i_{1}} \beta_{i_{2}} \ldots \beta_{i_{s}}$ into a finite number of irreducible Perron numbers, in finitely many ways, corresponds to a factorization of the beta-shift and of $\zeta_{\beta}(z)$ ([30] Theorem 4 and p. 298), therefore of $f_{\beta}(z)$.

The locus of the zeroes of the functions $f_{\beta}(z)$ for all real numbers $\beta>1$ is explored in Section 2 and discussed by comparison with older results. The geometry of this locus may give sufficient conditions for a Perron number not to be a Parry number (Section 3). In Section 3.2 we discuss the existence and the nature of the zeroes of $f_{\beta}(z)$ when $\beta>1$ is not a Parry number, trying to answer to (P2). In Section 4 we give partial results to (P1) when $\beta$ is a Parry number: we show how the Erdős-Turán approach [20] [28] refines Theorem 2.3 and correlates the set of Galois conjugates to the set of beta-conjugates. Examples of Pisot, Salem and Perron numbers are given for illustrating this correlation.

\section{Locus of zeroes}

Recall that a section of a power series is a polynomial obtained by truncating the "tail" of the power series. Let $S_{n}(z)=-1+\sum_{i=1}^{n} t_{i} z^{i}$ be the $n$ th-section of $f_{\beta}(z)$, for $n \geq 1$. 
Proposition 2.1. Let $\beta>1$ be a noninteger real number. The open disc $D\left(0, \frac{1}{\lfloor\beta\rfloor+1}\right)$ contains no zero of $f_{\beta}(z)$.

Proof. This is a consequence of Hurwitz Theorem (Marden [33] p. 4) and of the following Proposition applied to the sections of $f_{\beta}(z)$.

Proposition 2.2. Let $k \geq 1$ be an integer. Let $\kappa>0$ and $\left(\alpha_{i}\right)_{1 \leq i \leq k}$ be complex numbers such that $\left|\alpha_{i}\right|<\kappa(1 \leq i \leq k)$. Then the polynomial $-1+\alpha_{1} z+\alpha_{2} z^{2}+\ldots+$ $\alpha_{k} z^{k}$ has no root within the open disc $D\left(0, \frac{1}{\kappa+1}\right)$.

Proof. This is classical, see Barbeau [3] p. 188, ex. 30. See also Mignotte [35] and Theorem 3.4 in Yamamoto [51].

Let us improve Proposition 2.1.

Theorem 2.3. Let $m \geq 1$ be an integer. The region of the open unit disc in the complex plane "inside" the curve $\mathcal{C}_{m}$ defined by

$$
\mathcal{C}_{m}: \quad \frac{|z|^{2}}{1-|z|}=\left|\frac{-2}{m}+\frac{z(2-z)}{1-z}\right|
$$

is zerofree for $f_{\beta}(z)$ with $\beta>1$ any noninteger real number such that $m=\lfloor\beta\rfloor$. The curve $\mathcal{C}_{m}$ is symmetrical with respect to the real axis. If $m \geq 2$, it has two connected components: the first one $\mathcal{C}_{m}^{u n i t}$ defines a neighborhood of the unit circle, with a cusp at $z=1$, and the second one $\mathcal{C}_{m}^{\text {bubble }}$ a small neighborhood of $z=\beta^{-1}$, which contains the open interval $(1 /(m+1), 1 / m)$ of the real axis; the small neighborhood of $\beta^{-1}$ delimited by $\mathcal{C}_{m}^{\text {bubble }}$ only contains the zero $\beta^{-1}$ of $f_{\beta}(z)$. 
Proof. This is a consequence of Proposition 2.4, (2.1) being a reformulation of (2.3) since:

$$
\left|\frac{-2}{m}+\frac{z(2-z)}{1-z}\right|=\frac{1}{\lfloor\beta\rfloor}\left|\frac{-2+2(\lfloor\beta\rfloor+1) z-\lfloor\beta\rfloor z^{2}}{1-z}\right| .
$$

Figure 1 illustrates the case $m=2$. The small neighborhood of $\beta^{-1}$ delimited by $\mathcal{C}_{m}^{\text {bubble }}$ only contains one zero of $f_{\beta}(z)$ which is $z=1 / \beta$ itself by the following fundamental identity:

$$
-1+\sum_{i=1}^{\infty} t_{i} z^{i}=(-1+\beta z)\left(1+\sum_{j=1}^{\infty} T_{\beta}^{j}(1) z^{j}\right), \quad|z|<1
$$

where $T_{\beta}$ is the $\beta$-transform: $T_{\beta}^{1}=T_{\beta}:[0,1] \rightarrow[0,1], x \rightarrow \beta x \bmod 1, T_{\beta}^{j+1}(x)=$ $T_{\beta}\left(T_{\beta}^{j}(x)\right), j \geq 0$, and $T_{\beta}^{0}=I d$. Indeed, the zeros $\neq \beta^{-1}$ of $-1+\sum_{i=1}^{\infty} t_{i} z^{i}$ are those of $1+\sum_{j=1}^{\infty} T_{\beta}^{j}(1) z^{j}$ by $(2.2)$; but the power series $1+\sum_{j=1}^{\infty} T_{\beta}^{j}(1) z^{j}$ is a particular case of the power series with (real) coefficients in [0, 1] studied by Solomyak [45]. From [45] the zeroes are in a subset of $D(0,1)$ (called $\mathcal{G}$ in [45]) which does not contain any neighborhood of any element of the interval $(0,1)$. Hence the claim.

The zeros $\neq \beta^{-1}$ of $f_{\beta}(z)$ cluster near the unit circle in the region delimited by $\mathcal{C}_{m}^{\text {unit }}$

Proposition 2.4. Let $\beta>1$. Let $z$ be a zero of $f_{\beta}(z)$ in $0<|z|<1$. Then

$$
\frac{\lfloor\beta\rfloor|z|^{2}}{1-|z|} \geq\left|\frac{-2+2(\lfloor\beta\rfloor+1) z-\lfloor\beta\rfloor z^{2}}{1-z}\right|
$$

Proof. If $z$ is a zero of $f_{\beta}(z)$ in the unit open disc $D(0,1)$, then

$$
f_{\beta}(z)=-1+\lfloor\beta\rfloor z+\frac{\lfloor\beta\rfloor}{2} \sum_{k=2}^{\infty} z^{k}+h(z)
$$




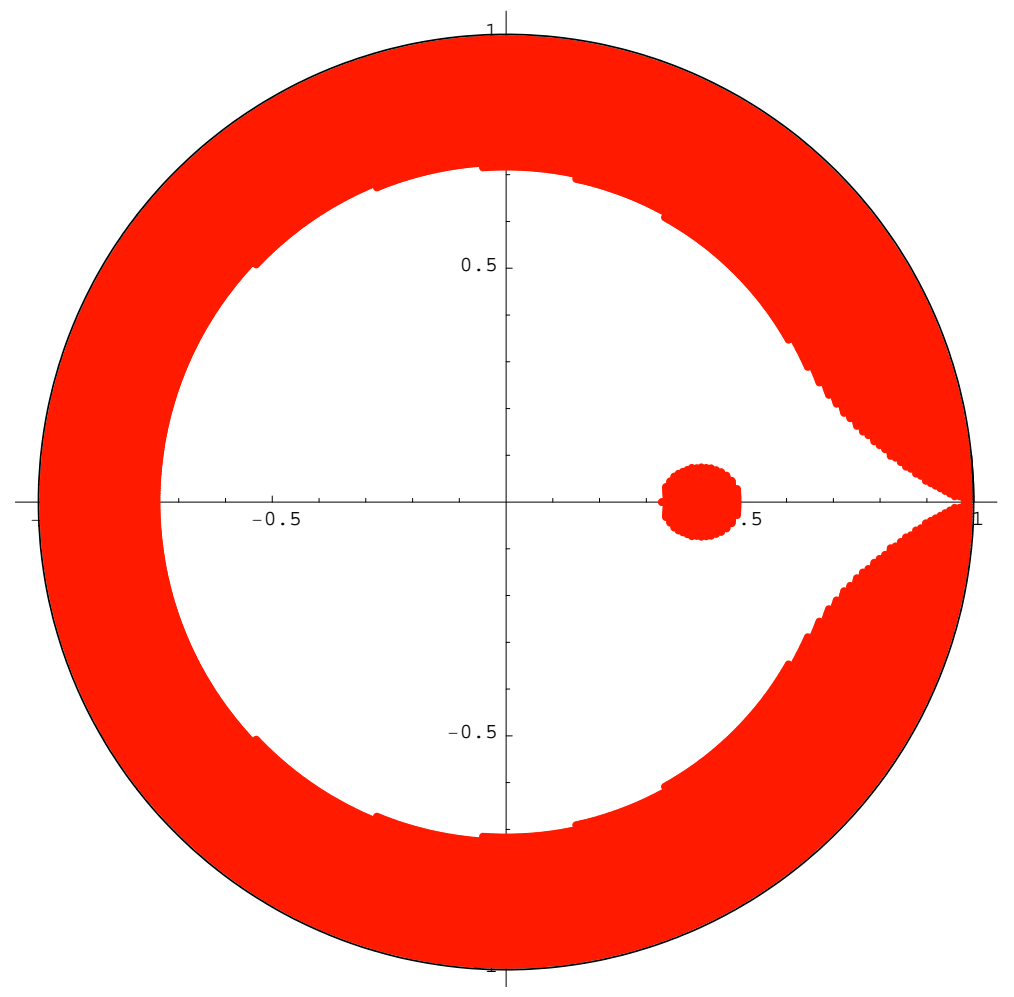

Figure 1. Zerofree region of the analytical function $f_{\beta}(z)$, delimited by the curve $\mathcal{C}_{m}$, for $\beta$ an algebraic number such that $m=\lfloor\beta\rfloor$. Here, $m=2$. 
with

$$
h(z)=\frac{\lfloor\beta\rfloor}{2} \sum_{k=2}^{\infty} u_{k} z^{k} \quad\left|u_{k}\right| \leq 1, \text { for all } k \geq 2,
$$

and

$$
|h(z)| \leq \frac{\lfloor\beta\rfloor}{2} \frac{|z|^{2}}{1-|z|}
$$

For $|z|<1$,

$$
f_{\beta}(z)=\frac{-2+2(\lfloor\beta\rfloor+1) z-\lfloor\beta\rfloor z^{2}}{2(1-z)}+h(z) .
$$

If $(2.3)$ is not satisfied, then $f_{\beta}(z)$ would be $\neq 0$.

The following Theorem holds true for all numeration bases $\beta$ in an open interval of the type $(1, C)$ with $1<C<+\infty$.

Theorem 2.5. For all $C>1$ the set of zeroes of the functions $f_{\beta}(z)$ where $\beta$ runs over $(1, C)$ lies within the closed ball

$$
|z| \leq 2\lfloor C\rfloor
$$

Proof. This comes from Marden [33] ex. 35.5, p 122 applied to the sections of $f_{\beta}(z)$ : indeed, for any polynomial section $a_{0}+a_{1} z^{n_{1}}+a_{2} z^{n_{2}}+\ldots+a_{k} z^{n_{k}}$ of $f_{\beta}(z)$ (with all $a_{i}$ s nonzero in $\left.\mathcal{A}_{\beta}\right)$, all its zeroes lie in the disk

$$
|z| \leq \max \left\{\left|\frac{a_{0}}{a_{1}}\right|^{1 / n_{1}},\left|\frac{2 a_{n_{j}}}{a_{n_{j+1}}}\right|^{\frac{1}{n_{j+1}-n_{j}}}\right\} \quad j=1,2, \ldots, k-1 .
$$

Here $\left|a_{0}\right|=1, n_{1}=1$ and $a_{1}=\lfloor\beta\rfloor$. Then the radius of this disk is

$$
\leq \max _{j=1, \ldots, k-1}\left|\frac{2 a_{n_{j}}}{a_{n_{j+1}}}\right|^{\frac{1}{n_{j+1}-n_{j}}} \leq \max _{j=1, \ldots, k-1}\left|\frac{2\lfloor\beta\rfloor}{1}\right|^{\frac{1}{n_{j+1}-n_{j}}} \leq 2\lfloor\beta\rfloor .
$$


We now conclude using Hurwitz Theorem ([33] p. 4) for the zeroes of the Taylor series $f_{\beta}(z)$

Therefore, in case $(\mathbf{D})(\mathrm{i})$ the zeroes $\neq \beta^{-1}$ of $f_{\beta}(z)$ lie between the curve $\mathcal{C}_{m}$ (resp. $\mathcal{C}_{m}^{\text {unit }}$ if $m \geq 2$ ) and the circle of radius $2(m+1)$ centred at the origin, for $m=\lfloor\beta\rfloor \geq 1$; in case $(\mathbf{D})\left(\right.$ ii) the zeroes $\neq \beta^{-1}$ of $f_{\beta}(z)$ lie between the curve $\mathcal{C}_{m}$ (resp. $\mathcal{C}_{m}^{\text {unit }}$ if $m \geq 2$ ) and the unit circle. The locus of all possible zeroes $\neq \beta^{-1}$ of $f_{\beta}(z)$ in $|z|<1$, for all $\beta>1$, is represented in Figure 1 in [45] (it has a spike on $\mathbb{R}^{-}$).

When $m$ goes to infinity the sequence of curves $\left(\mathcal{C}_{m}^{b u b b l e}\right)_{m \geq 1}$ shrinks to 0 while the sequence $\left(\mathcal{C}_{m}^{\text {unit }}\right)_{m \geq 1}$ shrinks monotonously towards the centre of the unit disc and converges to the limit curve

$$
\mathcal{C}_{\infty}^{\text {unit }}: \quad \frac{|z|}{1-|z|}=\left|\frac{2-z}{1-z}\right|
$$

This limit curve has a cusp at $z=1$. Denote by $\tau=\frac{1+\sqrt{5}}{2}$ the golden mean. Proposition 2.1 is improved as follows.

Proposition 2.6. The points $z_{0}=\frac{-1}{\tau}, z=1$ belong to $\mathcal{C}_{\infty}^{\text {unit }}$ and any $z \in \mathcal{C}_{\infty}^{\text {unit }}, z \neq$ $z_{0}, z \neq 1$, is such that

$$
\frac{1}{\tau}<|z|<1
$$

In particular, for every noninteger real number $\beta>1, \beta \neq \tau$, the zeros $\neq \beta^{-1}$ of $f_{\beta}(z)$ have a modulus $>1 / \tau$.

Proof. Corollary 4.2 in [21], Corollary 2.3 in [45]. 


\section{Beta-conjugates, Galois conjugates and dichotomy}

Let $\beta>1$ be a real number, $m=\lfloor\beta\rfloor$. The inverses of the zeroes $\neq \beta^{-1}$ of $f_{\beta}(z)$ lie within the curve which is the image of $\mathcal{C}_{\infty}^{\text {unit }}$ by the transformation $z \rightarrow 1 / z$ by Theorem 2.3. The following Proposition indicates the maximal possible thickening of the image of this curve when $\beta$ runs over a neighbourhood of infinity.

Proposition 3.1. Let $\epsilon$ be a fixed real number such that $0<\epsilon \leq 2$. Then for all real numbers $\beta$ such that $2 / \epsilon \leq\lfloor\beta\rfloor$, the inverses of the zeroes of $f_{\beta}(z)$ lie in

$$
\left\{z \in \mathbb{C}\left|\frac{1}{|z|(|z|-1)} \leq\right| \frac{2 z-1}{z(z-1)} \mid+\epsilon\right\} .
$$

Proof. The image of $\mathcal{C}_{m}$ (given by (2.1)) by $z \rightarrow 1 / z$ is the curve defined by

$$
\frac{1}{|z|(|z|-1)}=\left|\frac{-2}{m}+\frac{2 z-1}{z(z-1)}\right|, \quad m=\lfloor\beta\rfloor,
$$

and

$$
\left|\frac{-2}{m}+\frac{2 z-1}{z(z-1)}\right| \leq\left|\frac{2 z-1}{z(z-1)}\right|+\epsilon
$$

since $\frac{2}{[\beta]} \leq \epsilon$. We deduce (3.1).

The limit curve $\mathcal{C}_{\infty}^{\text {unit }}{ }^{-1}$ :

$$
\frac{1}{|z|-1}=\left|\frac{2 z-1}{z-1}\right|
$$

independent of $\beta$, obtained by allowing $\epsilon$ to zero and by multiplying by $|z|>1$, in (3.2), contains the domain $\Phi$ of the inverses of the zeroes $\neq \beta^{-1}$ of the functions $f_{\beta}(z)$, for all $\beta>1$, which was extensively studied in [45]. Figure 2 in [45] represents 
the boundary of the domain $\Phi$, which is claimed related to all Parry numbers $\beta>1$, but which is actually related to the whole collection of real numbers $\beta>1$ by the definition of $\mathcal{B}$ in [45], p. 478-9.

Whether the inverse of a Galois conjugate of an algebraic number $\beta>1$ is a zero or not of $f_{\beta}(z)$ receives a clear answer in the first case of $(\mathbf{D})$, not in the second case.

\subsection{The rational fraction case}

In this paragraph $f_{\beta}(z)$ is assumed to be a rational fraction according to $(\mathbf{D})(\mathrm{i})$, and $\beta$ is a Parry number, hence a Perron number. We have:

$$
f_{\beta}(z)=\frac{\left(1-z^{n+1}\right) U(z)+z^{m+1} V(z)}{1-z^{n+1}} .
$$

Since it cancels at $\beta^{-1}$ and is an element of $\mathbb{Q}[z]$, it cancels at the inverses of the Galois conjugates of $\beta$. Then the numerator is a multiple of $P_{\beta}^{*}(z)$, where $P_{\beta}^{*}(X)=X^{d} P_{\beta}(1 / X)$ is the reciprocal polynomial of the minimal polynomial $P_{\beta}(X)$ of $\beta(\operatorname{deg}(\beta)=d \geq 2)$. The function $f_{\beta}(z)$ takes the form

$$
f_{\beta}(z)=\frac{P_{\beta}^{*}(z) R_{\beta}^{*}(z)}{1-z^{n+1}}
$$

where $R_{\beta}^{*}(X) \in \mathbb{Q}[X]$ is the reciprocal polynomial of the complementary factor $R_{\beta}(X)$ of Boyd [14]

It seems difficult to establish an approximative equation of the boundary of $\Phi$. Therefore, the following simple geometric criterium may be useful. 
Proposition 3.2. If $\beta>1$ is a Perron number which admits one Galois conjugate outside $\mathcal{C}_{\infty}^{\text {unit }}{ }^{-1}$, given by (3.3), e.g. if

(i) one Galois conjugate of modulus is $>\tau\left(=\frac{1+\sqrt{5}}{2}\right)$, or if

(ii) a real conjugate is $>1$,

then $\beta$ is not a Parry number.

Proof. If we assume that $\beta$ is a Perron number with one Galois conjugate $\beta^{\prime}$ outside $\mathcal{C}_{\infty}^{\text {unit }}{ }^{-1}$ and that $\beta$ is a Parry number, the inverse $\beta^{-1}$ would be a zero of $f_{\beta}(z)$. The contradiction comes from the definition of $\mathcal{C}_{\infty}^{\text {unit }}$. In particular for (i), the case $\beta^{\prime}>\tau$ is impossible by Proposition 2.6; and for (ii), the case $1<\beta^{\prime}<\beta$ leads to a contradiction. Indeed

$$
f_{\beta}\left(\frac{1}{\beta^{\prime}}\right) \text { would be }=0, \quad \text { with } \frac{1}{\beta}<\frac{1}{\beta^{\prime}}<1
$$

but the zerofree region in $|z|<1$ delimited by $\mathcal{C}_{m}^{\text {unit }}$, with $m=\lfloor\beta\rfloor$, contains the open interval $\left(\beta^{-1}, 1\right)$.

See also Remark 7.2.23 in Lothaire [31] for a direct proof of (ii).

Proposition 3.2(i) is of course addressed to Perron numbers $>\tau$. For Perron numbers in the interval $(1, \tau)$ we have for instance the following result, which is related to the question of smallest Salem numbers [44].

Proposition 3.3. Assume that there exists a sequence of Salem numbers $\left(\beta_{i}\right)_{i \geq 1}$ which converges to 1 . Then there exists $i_{0} \geq 1$ such that

$$
i \geq i_{0} \Longrightarrow \beta_{i} \text { is not a Parry number. }
$$


Proof. By Theorem 5.1 in [21] there exists $\theta>0$ such that for every Parry number $\beta$ in $(1,1+\theta)$ there exists a constant $c>0$ such that the zeroes of $f_{\beta}(z)$ are all in the annulus

$$
\frac{1}{\beta}+\frac{2 \pi^{2}(\beta-1)}{|\log (\beta-1)|^{3}}+c\left(\frac{(\beta-1) \log |\log (\beta-1)|}{|\log (\beta-1)|^{4}}\right)<|z|<1 .
$$

Assume now that there exists $i$ large enough such that $\beta_{i}$ is simultaneous in $(1,1+$ $\theta)$ and is a Parry number. Then the inverses of the Galois conjugates of $\beta_{i}$ would all be in the open annulus defined by (3.5), and never on the unit circle. But $\lim _{j \rightarrow+\infty}$ $\operatorname{deg}\left(\beta_{j}\right)=+\infty$, and $\beta_{i}$ has $\operatorname{deg}\left(\beta_{i}\right)-2$ Galois conjugates on $|z|=1$. This is of course a contradiction.

The existence of a sequence of Salem numbers as in Proposition 3.3 is unlikely in the context of Lehmer's Conjecture [29] and Lind-Boyd's Conjecture [12] [40] which proposes explicitely the possible Perron polynomials for Perron numbers close to 1 .

\subsection{The second case: Hadamard type theorems, frequencies of digits}

In this paragraph $f_{\beta}(z)$ is assumed to admit the unit circle as natural boundary (D)(ii), and $\beta>1$ is not a Parry number.

Theorem 1 in Salem ([41] p. 161) implies that every power series $f_{\beta}(z)$ maps the open unit disc densely into the complex plane:

$$
\overline{f_{\beta}(D(0,1))}=\mathbb{C}
$$


This result is independent of Szegö's dichotomy theorem. Searching for beta-conjugates of $\beta$ can then be deduced from the establishment of new theorems which complete this result by showing that the onto property of power series $f_{\beta}(z)$ holds in $D(0,1)$ as well (putting apart the trivial zero $\left.\beta^{-1}\right)$. It is the Picard property ([50] for lacunary power series). Even though the Picard property is satisfied, new theorems are also needed to formulate the zeroes of $f_{\beta}(z)$, their number and their geometry.

There are almost no result in this direction. Let us mention the following consequence of a theorem of Fuchs [25]. To take into account the nonzero coefficients $t_{i}$ of $f_{\beta}(z)$, let us define the sequence $\left(a_{n_{q}}\right)_{q \geq 0}$ by extracting the nonzero digits from the sequence $t_{1} t_{2} t_{3} \ldots$ in the increasing order. Then

$$
f_{\beta}(z)=\sum_{q=0}^{+\infty} a_{n_{q}} z^{n_{q}} \quad \text { with } 0=n_{0}<1=n_{1}<n_{2}<n_{3}<\ldots
$$

and $a_{n_{0}}=-1\left(=t_{0}\right), a_{n_{1}}=\lfloor\beta\rfloor\left(=t_{1}\right)$, etc.

We say that $f_{\beta}(z)=\sum_{j=0}^{\infty} a_{n_{j}} z^{n_{j}}$ contains Hadamard gaps if there exists $\theta>0$ such that

$$
n_{q+1}-n_{q}>\theta n_{q} \quad \text { for all } q \geq 1
$$

For $\beta>1$ an algebraic number, in particular a Perron number, the existence of Hadamard gaps requires [49]

$$
1<\frac{\log (\mathrm{M}(\beta))}{\log (\beta)} \text { and } 0<\theta<\frac{\log (\mathrm{M}(\beta))}{\log (\beta)}-1
$$

where $\mathrm{M}(\beta)=a \prod_{i=0}^{d-1} \max \left\{1,\left|\beta^{(i)}\right|\right\}$ denotes the Mahler measure of $\beta$ (with $a$ the leading (positive) coefficient of the minimal polynomial $P_{\beta}(X)$ of $\left.\beta\right)$. 
Theorem 3.4. If $\beta>1$ is not a Parry number which is such that $f_{\beta}(z)=\sum_{j=0}^{+\infty} a_{n_{j}} z^{n_{j}}$ contains Hadamard gaps, then the number of beta-conjugates of $\beta$ is infinite.

Proof. It is a direct consequence of Theorem 1 in Fuchs [25].

The possibility of an infinite number of zeroes of $f_{\beta}(z)$ claimed by Theorem 3.4 is not surprising in the context of the following conjecture proposed in [21].

Conjecture 1. (Flatto, Lagarias, Poonen) The set of real numbers $\beta>1$ such that $f_{\beta}(z)$ has infinitely many zeros in the open unit disc has full Lebesgue measure.

This Conjecture is addressed to transcendental numbers, mostly, and does not say anything on the possibility of an infinite number of zeroes of $f_{\beta}(z)$ when $\beta$ is an algebraic number which is not a Parry number.

In the same way, the question whether the inverses of the Galois conjugates of $\beta$ are zeroes of $f_{\beta}(z)$ remains open. Proposition 3.2 answers partially, and negatively, to this question. For instance, $\beta=(5+\sqrt{5}) / 2$ is a quadratic Perron number which has a real conjugate $>1$ and therefore is not a Parry number. On the contrary, it is not because the Galois conjugates of a Perron number $\beta$ all lie in $\operatorname{int}(\Phi)$ that $\beta$ is a Parry number (for instance, the quadratic Perron number $\beta=(1+\sqrt{13}) / 2([45] \mathrm{p}$. 483)).

Remark 3.5. The assumptions of Theorem 3.4 are probably unrealistic because of the frequencies of the digits occuring in $d_{\beta}(1)$ by [36], known for almost all $\beta$ s. Indeed, Parry proved, for almost all $\beta>1$, that the frequency of the digit $\kappa \in \mathcal{A}_{\beta}$ in $d_{\beta}(1)=$ 
$0 . t_{1} t_{2} t_{3} \ldots$ exists by the ergodic theorem of Birkhoff and that it is equal to

$$
= \begin{cases}\nu_{\beta}\left[\frac{\kappa}{\beta}, \frac{\kappa+1}{\beta}\right) & \text { if } \quad \kappa \in\{0,1, \ldots,\lfloor\beta\rfloor-1\} \\ \nu_{\beta}\left[\frac{\lfloor\beta\rfloor}{\beta}, 1\right) & \text { if } \quad \kappa=\lfloor\beta\rfloor\end{cases}
$$

where $\nu_{\beta}$ is the unique ergodic measure on $[0,1]$ associated with the $\beta$-shift; it is equivalent to the Lebesgue measure. But, under the assumptions of Theorem 3.4, the frequency of the digit 0 in $d_{\beta}(1)$ is 1 , and the frequencies of the other digits $\neq 0$ in this $\beta$-expansion are zero. This is impossible since all the intervals $\left[\frac{\kappa}{\beta}, \frac{\kappa+1}{\beta}\right), \kappa=$ $1,2, \ldots,\lfloor\beta\rfloor-1$ are of Lebesgue measure $\frac{1}{\beta}>0$. We deduce that the existence of Hadamard gaps almost never occurs (see also Schmeling [42]). But Theorem 3.4 is addressed to real numbers $\beta>1$ and is questionable for algebraic numbers $\beta>1$, whose set is countable. Therefore it has still to be proved that the set of algebraic numbers, in particular Perron numbers, which satisfy the assumptions of Theorem 3.4 is not empty.

Let us recall the classification of the numeration bases introduced by BertandMathis, as cited in Blanchard [11] [49]. They are based on the gaps of $d_{\beta}(1)$ and on the language $\mathcal{L}\left(S_{\beta}\right)$ of the $\beta$-shift:

$$
\begin{aligned}
& \mathrm{C}_{1}=\left\{\beta>1 \mid d_{\beta}(1) \text { is finite }\right\} . \\
& \mathrm{C}_{2}=\left\{\beta>1 \mid d_{\beta}(1) \text { is ultimately periodic but not finite }\right\} . \\
& \mathrm{C}_{3}=\left\{\beta>1 \mid d_{\beta}(1) \text { is infinite, contains bounded strings of } 0^{\prime} \mathrm{s}\right. \text {, but is not } \\
&\text { ultimately periodic }\} .
\end{aligned}
$$


$\mathrm{C}_{4}=\left\{\beta>1 \mid d_{\beta}(1)\right.$ does not contain some words of $\mathcal{L}\left(S_{\beta}\right)$, but contains strings of $0^{\prime}$ s with unbounded length\}.

$\mathrm{C}_{5}=\left\{\beta>1 \mid d_{\beta}(1)\right.$ contains all words of $\left.\mathcal{L}\left(S_{\beta}\right)\right\}$

The set of Parry numbers is $C_{1} \cup C_{2}$. The set of Perron numbers which are not Parry numbers is conjecturally included in $C_{3}$.

\section{Erdős-Turán approach}

\subsection{Uniform clustering near the unit circle}

The Erdős-Turán approach [20] allows a more accurate description of the zeroes of the functions $f_{\beta}(z), \beta>1$, which have a tendancy to cluster uniformly near the unit circle, in the sense of Weyl [28]. We will apply it by using the following theorem.

Theorem 4.1. Let $\beta>1$ be a real number. If the roots of

$$
\sum_{j=0}^{k} a_{j} z^{j}, \quad\left|a_{j}\right| \in\{0,1,2, \ldots,\lfloor\beta\rfloor\}, a_{0} a_{k} \neq 0,
$$

are denoted by

$$
z_{\nu}=r_{\nu} e^{i \phi_{\nu}}
$$

then there exist two constants $C_{1}, C_{2}>0$, such that

(i) for every angular sector $0 \leq \alpha<\eta \leq 2 \pi$,

$$
\left|\frac{1}{k} \sum_{\alpha \leq \phi_{\nu} \leq \eta} 1-\frac{\eta-\alpha}{2 \pi}\right|^{2}<C_{1} \operatorname{discrep}(k)
$$


(ii) for $0<\rho<1$,

$$
\left(1-\frac{\#\left\{z_{\nu}|1-\rho \leq| z_{\nu} \mid \leq \frac{1}{1-\rho}\right\}}{k}\right) \leq C_{2} \frac{1}{\rho} \operatorname{discrep}(k),
$$

with

$$
\operatorname{discrep}(k):=\frac{\left.\log \left(\sum_{j=0}^{k}\left|a_{j}\right|\right)\right)-\frac{1}{2} \log \left|a_{0}\right|-\frac{1}{2} \log \left|a_{k}\right|}{k}
$$

and the function discrep $(k)$ tends to zero when $k$ tends to infinity.

Proof. (i) is a consequence of Theorem I in Erdös-Turán [20], and (ii) of Theorem 3 in [28]. Since $a_{0} a_{k} \neq 0$ and $\left|a_{j}\right| \leq\lfloor\beta\rfloor$ for $j=0,1, \ldots, k$, we deduce

$$
\operatorname{discrep}(k) \leq \frac{\log ((1+k)\lfloor\beta\rfloor))}{k} \rightarrow 0, \quad k \rightarrow+\infty .
$$

Indeed, Theorem 4.1 can be applied either to

- the numerator $P_{\beta}^{*}(z) R_{\beta}^{*}(z)$ of $f_{\beta}(z)$ (see (3.4)) in the first case $(\mathbf{D})(\mathrm{i})$, or to

- the collection of the sections of $f_{\beta}(z)$, in the second case (D)(ii), since the zeroes of the analytical function $f_{\beta}(z)$ are the accumulation points of the set of zeroes of the sections $S_{n}(z)=-1+\sum_{i=1}^{n} t_{i} z^{i}$ of $f_{\beta}(z)$, and conversely, by Hurwitz's Theorem ([33], p. 4).

We will report elsewhere the second case and will develop the first case on examples in the next Subsections. The constants $C_{1}$ and $C_{2}$ are computed in [20] [28]: $C_{1}=$ $16^{2}, C_{2}=2$, for general polynomials and can be optimized, as well as the discrepancy function [10] [26] [34]. 
Theorem 4.1 is an interesting tool for detecting the existence of the beta-conjugates of an algebraic number $\beta$ and for understanding their distribution near the unit circle. This can be seen as follows: first it is easy to check that the moduli of the coefficients of the polynomial $P_{\beta}^{*}(z) R_{\beta}^{*}(z)$, as given by (3.4), are all in the alphabet $\mathcal{A}_{\beta}$, so that we can apply Theorem 4.1. Then we fix the angular opening $\eta-\alpha$ of the sector

$$
\mathcal{T}_{\alpha, \eta}:=\{z \in \mathbb{C} \mid \alpha \leq \arg z \leq \eta\}
$$

We now rotate $\mathcal{T}_{\alpha, \eta}$ around the origin. This means that we apply (4.1) to the sector $\mathcal{T}_{\alpha+x, \eta+x}$, for any $x \in[0,2 \pi]$. Since, for any $x \in[0,2 \pi] \eta-\alpha=(\eta+x)-(\alpha+x)$, we should have:

$$
\left|\frac{1}{k} \sum_{\alpha+x \leq \phi_{\nu} \leq \eta+x} 1-\frac{\eta-\alpha}{2 \pi}\right|^{2}<C_{1} \operatorname{discrep}(k) \quad \text { for all } x \in[0,2 \pi] .
$$

Then the strategy consists in chosing an opening $\eta-\alpha$ of $\mathcal{T}_{\alpha, \eta}$ which is canonically linked to the angular distribution of the Galois conjugates of $\beta$, and to use the counting measure $x \rightarrow \frac{1}{k} \sum_{\alpha+x \leq \phi_{\nu} \leq \eta+x} 1$ of (4.4) to detect, in this rotating sector, the beta-conjugates of $\beta$ in the (angular) holes left by the Galois conjugates, these holes corresponding to a finite number of values of $x$. This process counts the betaconjugates with a possible multiplicity which is induced by the geometry of the Galois conjugates. A natural possibility for the opening angle $\eta-\alpha$, which depends upon the asymptotic expression of the discrepancy ( $=$ rhs of $(4.1))$ when $k$ goes to infinity, is of the form $C_{3} \sqrt{\frac{k}{\log k}}$ where $C_{3}$ is a constant. 
The remarkable point is that the inequality (4.1) is addressed to the union of the collection of zeroes which arise from the Galois conjugate and of the collection of beta-conjugates, then establishing a correlation between these two sets of zeroes.

The value of the discrepancy controls this angular counting process: the smaller it is, the better is the angular distribution of the roots of $P_{\beta}^{*}(z) R_{\beta}^{*}(z)$. This discrepancy tends to zero when the degree $k$ tends to infinity as (4.3).

\subsection{Examples}

The Perron numbers $\beta>1$ the beta-conjugates of which are studied by the ErdösTurán approach are taken among families of Perron numbers indexed by one integer (Subsections 4.2.1 to 4.2.3) or by several integers (Subsection 4.2.4). In each case the discrepancy, which controls the inequality (4.1), goes to zero when the degree of $\beta$ increases ( $\beta$ running in a given family).

4.2.1 Selmer's Perron numbers in the class $C_{1}$ Let us consider the dominant $\operatorname{root} \beta=\beta_{d} \in(1,2)$ of the polynomial $X^{d+1}-X^{d}-1$ for $d \geq 4$ an integer. These Perron numbers $\beta$ were studied by Selmer [43]. The minimal polynomial $P_{\beta}(X)$ of $\beta$ is equal to $X^{d+1}-X^{d}-1$ if and only if $d \not \equiv 4(\bmod 6)$. If $d \equiv 4(\bmod 6)$ then, writing $d=6 q+4$, the minimal polynomial $P_{\beta}(X)$ of $\beta$ is the quotient of $X^{d+1}-X^{d}-1$ by $X^{2}-X+1$; in this case, the degree of $\beta$ is $d-1$ and the number of Galois conjugates of $\beta$ inside the open unit disc $D(0,1)$ is $2(1+2 q)$, resp. outside the closed unit disc, 
is $1+2 q$ (including $\beta$ ). The Rényi $\beta$-expansion of unity is

$$
d_{\beta}(1)=0.10^{d-1} 1
$$

in both cases, which is finite, what provides a sequence of Perron numbers $\left(\beta_{d}\right)$ in $C_{1}$. We have: $\lim _{d \rightarrow \infty} \beta_{d}=1$. In both cases, the corresponding analytical function $f_{\beta}(z)$ is $-1+z+z^{d+1}$. If $d \equiv 4(\bmod 6), f_{\beta}(z)$ is equal to the product of $\phi_{6}(z)$ by $-1+z^{2} \varphi(z)$ where $\varphi(z)$ is a product of cyclotomic polynomials (Table 1 ). Recall that the $n$th cyclotomic polynomial is $\phi_{n}(X):=\prod_{k}\left(X-e^{2 i \pi k / n}\right)$ where $k$ runs over all (strictly) positive integers less than $n$ that are relatively prime to $n(n \geq 2)$. Its zeros are the beta-conjugates $-j,-j^{2}$ (i.e. $\left.e^{-i \pi / 3}, e^{i \pi / 3}\right)$, roots of $\phi_{6}(z)$, and the inverses of the Galois conjugates of $\beta$ (including $\beta^{-1}$ ).

The "angular" spaces (holes) left at $\operatorname{Re}(z)=1 / 2$ by the Galois conjugates are occupied by the two beta-conjugates in order to satisfy the regularity condition of the distribution of zeros of the sections of $f_{\beta}(z)$ by Theorem 4.1. The two beta-conjugates $-j,-j^{2}$ are independant of $d$. If $d \not \equiv 4(\bmod 6), \beta$ has no beta-conjugate, the angular distribution of the Galois conjugates satisfying by itself the regularity condition (4.1) of Theorem 4.1. 
Table 1: Function $f_{\beta}(z)$ for some Selmer's Perron numbers $\beta_{d}$ such that $d \geq 4, d \equiv$ $\underline{4(\bmod 6) .}$ The beta-conjugates of $\beta_{d}$ are the $(d$-independent $)$ roots of $\phi_{6}(z)$.

\begin{tabular}{|c|c|c|}
\hline$d$ & $\beta$ & $f_{\beta}(z)$ \\
\hline 4 & $1.32471 \ldots$ & $\phi_{6}(z)\left(-1+z^{2} \phi_{2}(z)\right)$ \\
\hline 10 & $1.18427 \ldots$ & $\phi_{6}(z)\left(-1+z^{2} \phi_{2}(z) \phi_{18}\right)$ \\
\hline 16 & $1.13390 \ldots$ & $\phi_{6}(z)\left(-1+z^{2} \phi_{2}(z) \phi_{10}(z) \phi_{30}(z)\right)$ \\
\hline 22 & $1.10695 \ldots$ & $\phi_{6}(z)\left(-1+z^{2} \phi_{2}(z) \phi_{14}(z) \phi_{42}(z)\right)$ \\
\hline 28 & $1.08986 \ldots$ & $\phi_{6}(z)\left(-1+z^{2} \phi_{2}(z) \phi_{18}(z) \phi_{54}(z)\right)$ \\
\hline 34 & $1.07794 \ldots$ & $\phi_{6}(z)\left(-1+z^{2} \phi_{2}(z) \phi_{22}(z) \phi_{66}(z)\right)$ \\
\hline 40 & $1.06909 \ldots$ & $\phi_{6}(z)\left(-1+z^{2} \phi_{2}(z) \phi_{26}(z) \phi_{78}(z)\right)$ \\
\hline 46 & $1.06222 \ldots$ & $\phi_{6}(z)\left(-1+z^{2} \phi_{2}(z) \phi_{18}(z) \phi_{10}(z) \phi_{30}(z) \phi_{90}(z)\right)$ \\
\hline$\vdots$ & $\vdots$ & $\vdots$ \\
\hline 64 & $1.04844 \ldots$ & $\phi_{6}(z)\left(-1+z^{2} \phi_{2}(z) \phi_{18}(z) \phi_{14}(z) \phi_{42}(z) \phi_{126}(z)\right)$ \\
\hline 70 & $1.04522 \ldots$ & $\phi_{6}(z)\left(-1+z^{2} \phi_{2}(z) \phi_{46}(z) \phi_{138}(z)\right)$ \\
\hline 76 & $1.04244 \ldots$ & $\phi_{6}(z)\left(-1+z^{2} \phi_{2}(z) \phi_{10}(z) \phi_{30}(z) \phi_{50}(z) \phi_{150}(z)\right)$ \\
\hline$\vdots$ & $\vdots$ & $\vdots$ \\
\hline 604 & $\begin{array}{l}1.00802 \ldots \\
\vdots\end{array}$ & $\begin{array}{l}\phi_{6}(z)\left(-1+z^{2} \phi_{2}(z) \phi_{18}(z) \phi_{134}(z) \phi_{402}(z) \phi_{1206}(z)\right) \\
\vdots\end{array}$ \\
\hline
\end{tabular}

4.2.2 Bassino's infinite family of cubic Pisot numbers Let $k \geq 2$ be an integer.

Let $\beta=\beta_{k}$ be the dominant root of the minimal polynomial

$$
P_{\beta}(X)=X^{3}-(k+2) X^{2}+2 k X-k .
$$




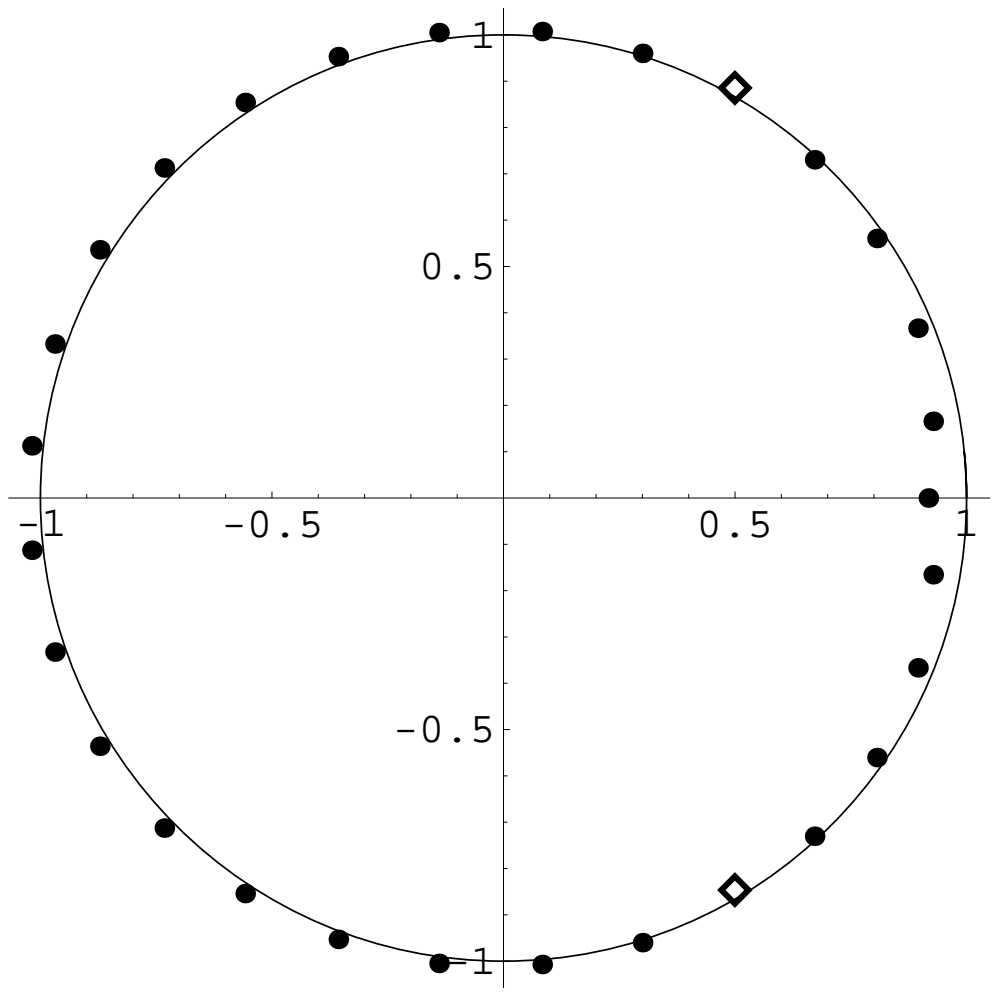

Figure 2. Inverses of the Galois conjugates $(\bullet)$ and of the beta-conjugates $(\diamond)$ of the Perron number $\beta=1.08986 \ldots$ dominant root of $X^{29}-X^{28}-1$. The beta-conjugates $-j,-j^{2}$ of $\beta$ lie within the angular holes left by the Galois conjugates. 
We have: $k<\beta_{k}<k+1$ and $\lim _{k \rightarrow+\infty}\left(\beta_{k}-k\right)=0$. The set $\left(\beta_{k}\right)$ is a subset of the class $\mathrm{C}_{1}$ [4]. The length of $d_{\beta_{k}}(1)$ is $2 k+2$ and

$$
f_{\beta_{k}}(z)=-1+k z+\sum_{i=2}^{k-1}\left((i-1) z^{i}+(k-i+1) z^{k+i+1}\right)+k z^{k}+z^{k+1}+k z^{2 k+2} .
$$

The case $k=30$ is illustrated in Figure 3. Let us write $P_{\beta}(X)=(X-\beta)(X-\alpha)(X-\bar{\alpha})$ where $\Im m \alpha>0$. The large "angular" sector $\{z \in \mathbb{C} \mid \arg \alpha<\arg z<2 \pi-\arg \alpha\}$ left free between the two Galois conjugates of $\beta_{k}$ is occupied regularly by the betaconjugates. It suffices to take a sector of opening angle equal to $\arg \alpha$ and rotate it anticlockwise from $\alpha$ to detect two beta-conjugates approximately at each integer multiple of $\arg \alpha$, within this sector. The multiplicity of 2 arises from the fact that $\alpha$ is very close to the first beta-conjugate of $\beta$, with a dedoubling in the vicinity of $z=-1$. The beta-conjugates appear as roots of products of cyclotomic polynomials for each $k$, of a certain type, though we have no general proof of this fact.

4.2.3 Boyd's infinite families of Pisot and Salem numbers A great number of examples of beta-conjugates of Pisot numbers and Salem numbers $\beta$, when $d_{\beta}(1)$ is eventually periodic, can be found in several publications of Boyd [13] [14] [15] [16]. Let us reformulate the following two results of Boyd in view of examplifying Theorem 4.1. 


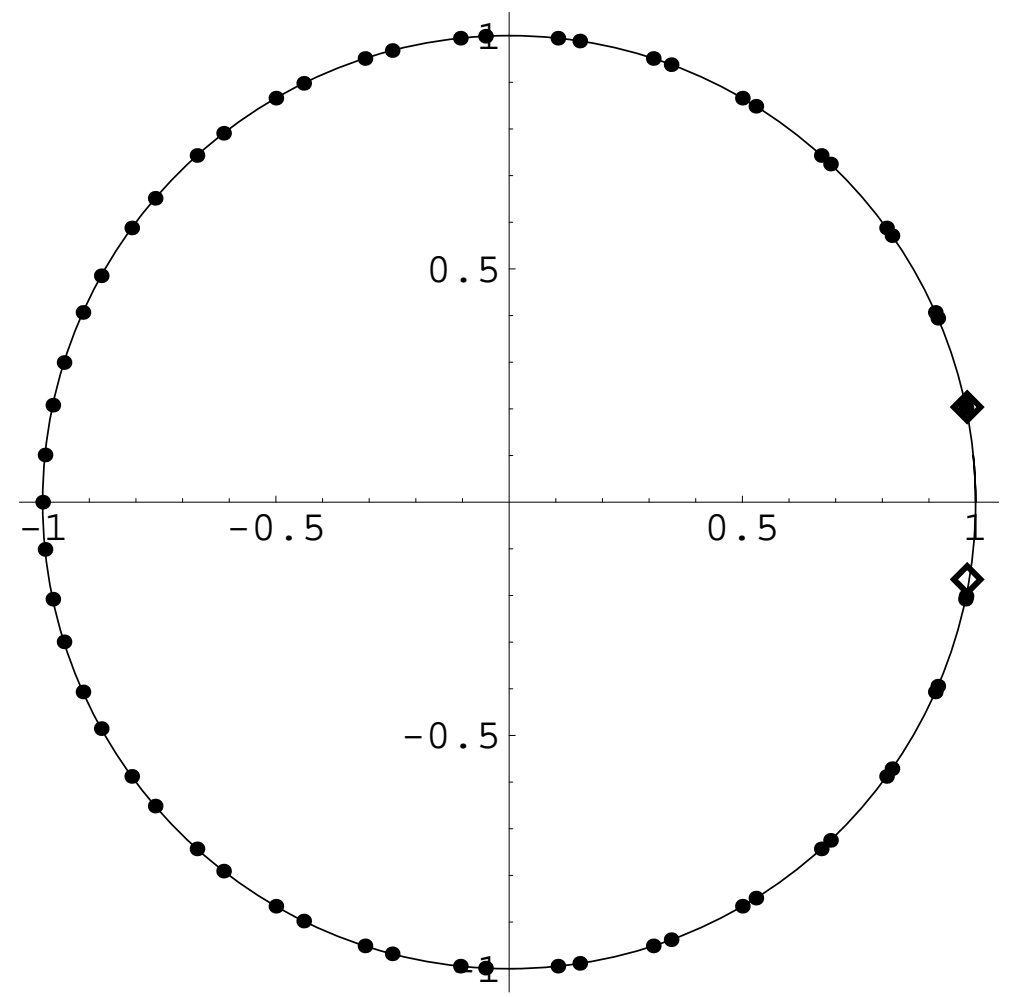

Figure 3. Galois conjugates $(\diamond)$ and beta-conjugates $(\bullet)$ of the cubic Pisot number $\beta=30.0356 \ldots$, dominant root of $X^{3}-32 X^{2}+60 X-30$. The beta-conjugates are the roots of $\phi_{2}(X) \phi_{3}(X) \phi_{6}(X) \phi_{10}(X) \phi_{10}(-X) \phi_{30}(X) \phi_{30}(-X) \phi_{31}(X)$. 
Proposition 4.2. Let $\beta=\beta_{k}$, with $k \geq 3$ an integer, be the Pisot number, dominant root of the minimal polynomial

$$
P_{\beta}(X)= \begin{cases}\frac{V_{k}(X)}{X-1} & \text { if } k \text { even } \\ \frac{V_{k}(X)}{X^{2}-1} & \text { if } k \text { odd }\end{cases}
$$

where

$$
\begin{aligned}
V_{k}(X) & =X^{6 k+1}(X-2)+X^{5 k}\left(X^{2}-X+1\right)-X^{4 k+1}(X-1) \\
& -X^{3 k}\left(X^{2}-2 X+1\right)-X^{2 k}+X^{k}\left(X^{2}-X+1\right)-X+1 .
\end{aligned}
$$

Then

$$
d_{\beta}(1)=0 .\left(1^{k-1} 010^{k-2} 10^{k+1} 1^{k-1} 0^{k} 1\right)\left(1^{k-2} 010^{k-1} 1^{k-1} 010^{k-2} 10^{k+1}\right)^{\omega}
$$

for which the preperiod is of length $5 k+1$ and the period of length $5 k$, and

$$
f_{\beta}(z)=\frac{P_{\beta}^{*}(z) R_{\beta}^{*}(z)}{1-z^{5 k}} \quad \text { with } R_{\beta}^{*}(X)= \begin{cases}X^{4 k}-X^{3 k}-1 & k \text { even } \\ (1+X)\left(X^{4 k}-X^{3 k}-1\right) & k \text { odd } .\end{cases}
$$

Proof. This is Proposition 5.4 in Boyd [14] (Figure 4 with $k=11$ ).

In Proposition $4.2, \beta_{k}<2$ for all $k \geq 3$ and $\lim _{k \rightarrow+\infty} \beta_{k}=2$; the beta-conjugates of $\beta$ are simple roots of the complementary factor.

Proposition 4.3. Let $\beta=\beta_{a}$ be the Salem number of degree 6 , dominant root of the minimal polynomial

$$
P_{\beta}(X)=X^{6}+(-a+1) X^{5}-a X^{4}-2 a X^{3}-a X^{2}+(-a+1) X+1
$$

with $a \geq 4$ an integer. Then, 


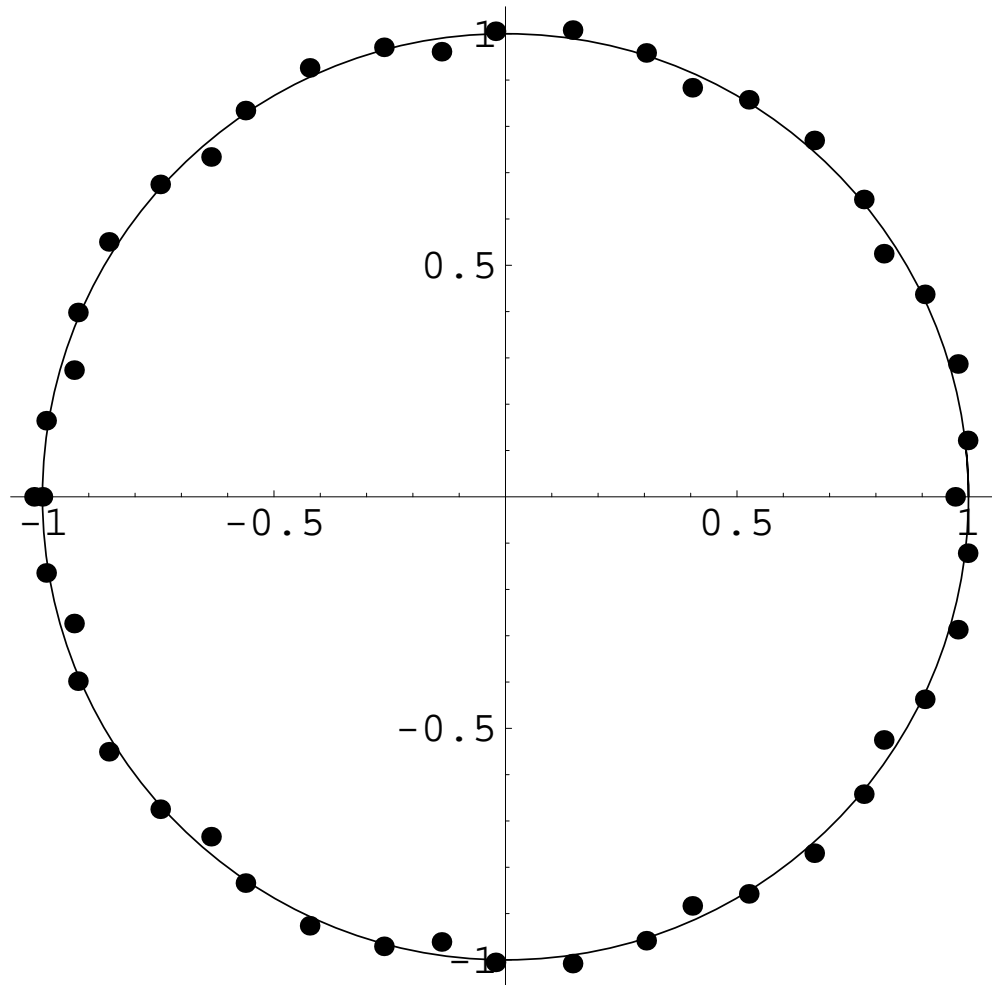

Figure 4. Beta-conjugates of the Pisot number $\beta=\beta_{11}=1.99926 \ldots$, of degree 66 , dominant root of $P_{\beta_{11}}(X)$ (Proposition 4.2). They are the roots of $(1+X)\left(X^{44}+\right.$ $\left.X^{11}-1\right)$. The beta-conjugates are near the unit circle between the Galois conjugates (not represented) so that their union is regularly angularly distributed in the sense of Theorem 4.1 . 


$$
d_{\beta}(1)=a 1(a-2) a 1
$$

$((a-2) 0 a 00(a-2) 11(a-2) 00 a 0(a-2) 20(a-4) 31(a-4) 20(a-2) 2(a-2) 02(a-4) 13(a-4) 02)^{\omega}$

for which, for all integer $a \geq 4$, the preperiod is of length 5 and the period of length

33 , and

$$
f_{\beta}(z)=\frac{P_{\beta}(z) R_{\beta}^{*}(z)}{1-z^{33}} \quad \text { with } R_{\beta}^{*}(X)=\phi_{2}(X) \phi_{6}(X)\left(X^{9}-X^{7}-1\right) \phi_{33}(X) .
$$

Proof. This is Proposition 4.3 in [15] (Figure 5).

As in the above example the set of beta-conjugates of $\beta_{a}$ is independent of the integer $a \geq 4$, so that this set does not characterize the Salem number $\beta_{a}$.

In the example of Figure 5 (with $a=4$ ), the counting process in small (rotating) sectors takes into account the multiplicity of 3 , which is the number of real roots $>0$ of $f_{\beta}(z)$.

4.2.4 Confluent Parry numbers They were studied by Frougny [22] and Bernat [5] [6]. By definition, a confluent Parry number $\beta$ is a Parry number which satisfies

$$
d_{\beta}(1)=0 . k_{1}^{d-1} k_{2}
$$

for some integers $d \geq 2, k_{1} \geq k_{2} \geq 1$. Such Parry numbers form a subclass of Perron numbers $\left(\beta_{d, k_{1}, k_{2}}\right)$ in $\mathrm{C}_{1}$. For such a Perron number, we have

$$
f_{\beta}(z)=-1+k_{1}\left(z+z^{2}+\ldots+z^{d-1}\right)+k_{2} z^{d}
$$

By Theorem 2 in Brauer [17] the polynomial

$$
X^{d} f_{\beta}(1 / X)
$$




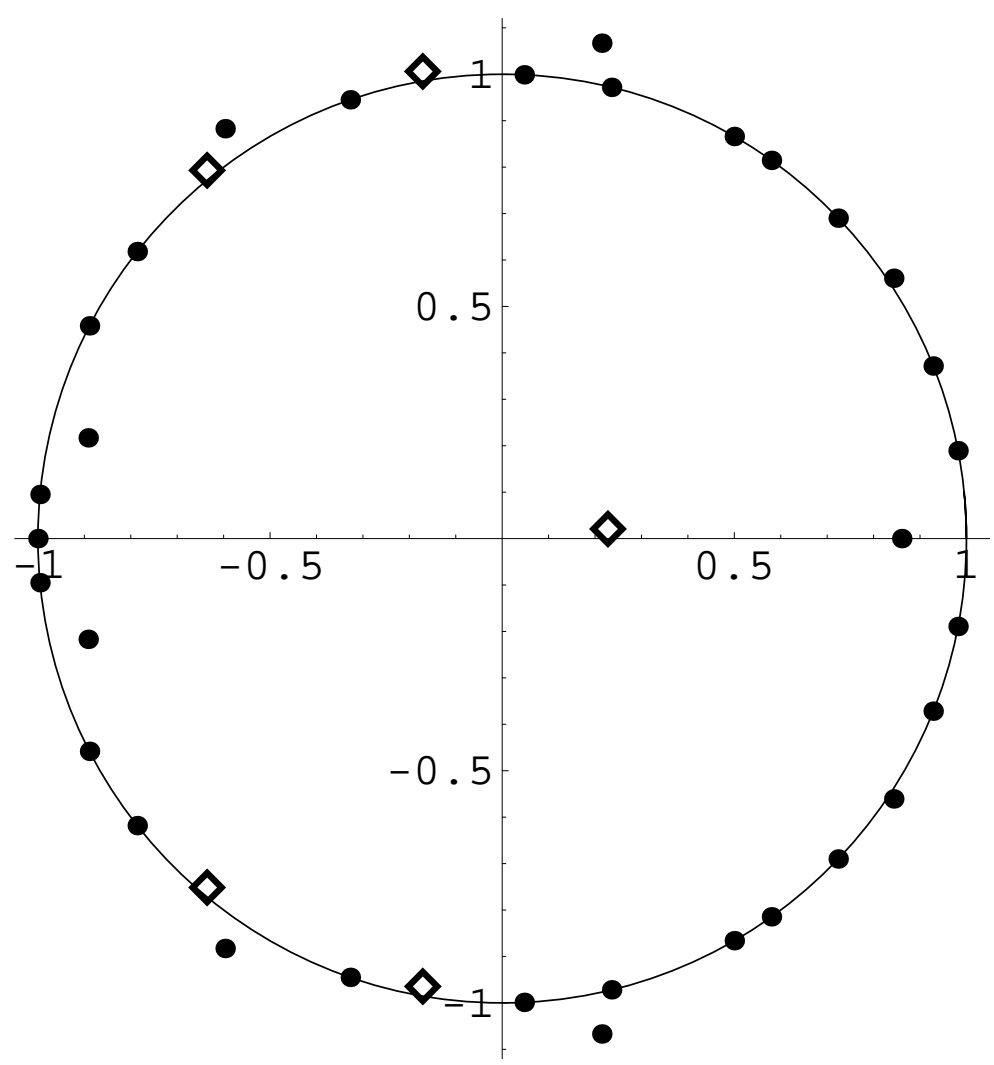

Figure 5. $a$-independent beta-conjugates $(\bullet)$ of the Salem number $\beta=\beta_{a}$, for $a \geq 4$ an integer, dominant root of $X^{6}-(a-1) X^{5}-a X^{4}-2 a X^{3}-a X^{2}-(a-1) X+1$. They are the roots of $\phi_{2}(X) \phi_{6}(X)\left(X^{9}+X^{2}-1\right) \phi_{33}(X)$. The beta-conjugates lie near the unit circle "between" the Galois conjugates $(\diamond$, represented here with $a=4)$ so that their union is regularly distributed in the sense of Theorem 4.1 
is irreducible for all integers $d \geq 2, k_{1} \geq k_{2} \geq 1$. This implies that the minimal polynomial $P_{\beta}(X)$ of $\beta$ is equal to $-X^{d} f_{\beta}(1 / X)$. This means that $\beta$ has no betaconjugate and therefore that the angular distribution of its Galois conjugates is very regular in the sense of Theorem 4.1. This is illustrated in Figure 6 for $k_{1}=9, k_{2}=4$ and $d=40$.

Such Parry numbers are Pisot numbers by the following theorem of Brauer: it suffices to consider

$$
(1-X) X^{d} f_{\beta}(1 / X)=X^{d+1}-\left(1+k_{1}\right) X^{d}+\left(k_{1}-k_{2}\right) X+k_{2} .
$$

Theorem 4.4. Let $z_{0}$ be a point on the unit circle which has the same argument as $-a_{1}$. Assume that the coefficients of the polynomial

$$
f(X)=X^{n}+a_{1} X^{n-1}+\ldots a_{n}, \quad a_{i} \in \mathbb{C},
$$

satisfy

$$
\left|a_{1}\right|=1+\left|a_{2}\right|+\left|a_{3}\right|+\ldots+\left|a_{n}\right| .
$$

Then the point $z_{0}$ may be a root of $f(z)$; at most one root different from $z_{0}$ lies in the exterior of the closed unit disc or on the unit circle while the others are in the open unit disc.

Proof. Brauer [17] p 254. 


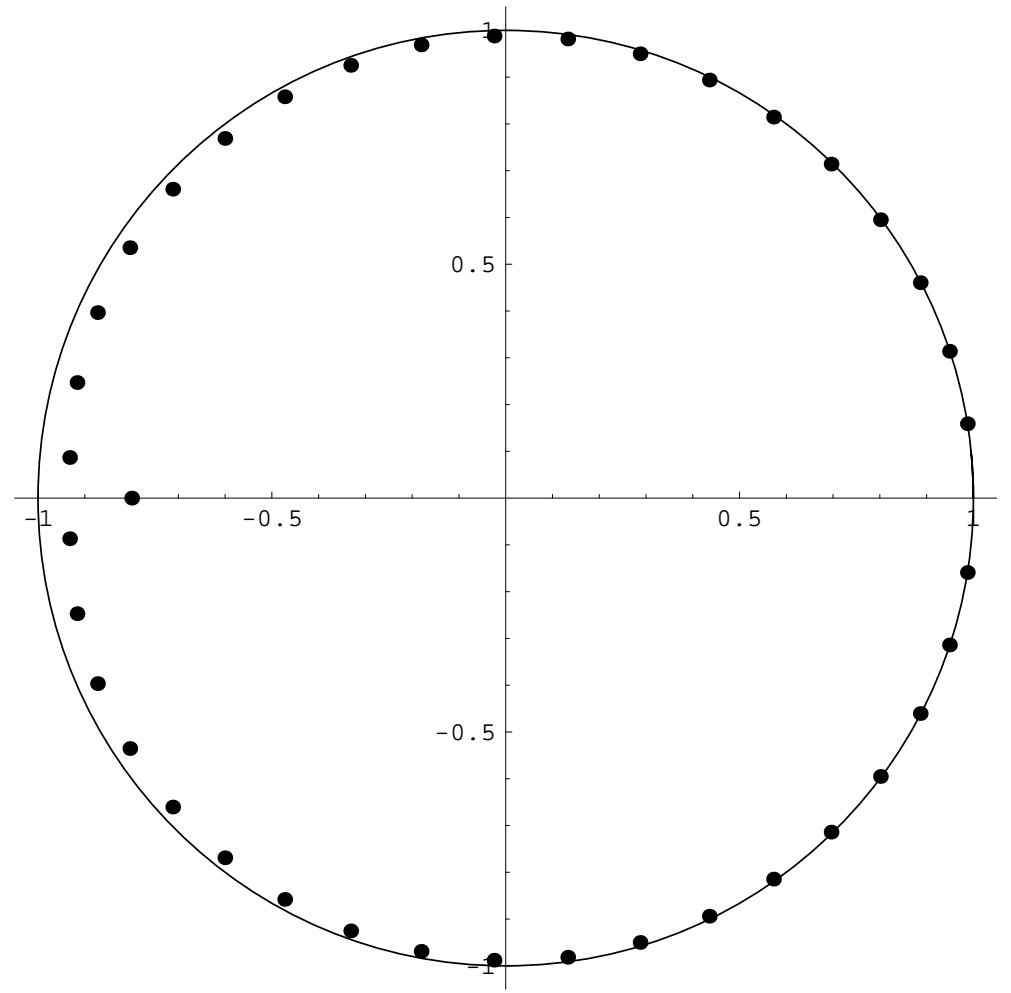

Figure 6. Galois conjugates $(\neq \beta)$ of the Perron number $\beta=9.999 \ldots$, dominant root of $P_{\beta}(X)=X^{40}-9 \sum_{i=1}^{39} X^{i}-4$, and confluent Parry number ( $\beta$ is a Pisot number). The absence of beta-conjugates is controlled by the great regularity of the angular distribution of the Galois conjugates, by Theorem 4.1 . 


\section{Acknowledgements}

The author is indebted to Pierre Liardet and to the anonymous referee for valuable comments and discussions.

\section{References}

[1] S. Akiyama, Cubic Pisot numbers with finite beta expansions, in Algebraic Number Theory and Diophantine Analysis (Graz, 1998), ed. by F. Halter-Koch and R.F. Tichy, de Gruyter, Berlin (2000), 11-26.

[2] G. Barat, V. Berthé, P. Liardet and J. Thuswaldner, Dynamical Directions in Numeration, Annales Inst. Fourier 56 (2006), 1987-2092.

[3] E.J. Barbeau, Polynomials, Springer (1989).

[4] F. Bassino, Beta-expansions for cubic Pisot numbers, Lecture Notes in Computer Sci. 2286 (2002), 141-152.

[5] J. Bernat, Arithmetic automaton for Perron numbers, Discr. Math. \& Theor. Comp. Sci. (2005), 1-29.

[6] J. Bernat, About a class of simple Parry numbers, J. of Automata, Languages and Combinatorics (2005).

[7] A. Bertrand-Mathis, Développements en base Pisot et répartition modulo 1, C. R. Acad. Sci. Paris, Série A, t. 285, (1977), 419-421. 
[8] A. Bertrand-Mathis, Développements en base $\theta$ et répartition modulo 1 de la suite $\left(x \theta^{n}\right)$, Bull. Soc. Math. Fr. 114 (1986), 271-324.

[9] A. Bertrand, Nombres de Perron et problèmes de rationnalité, Astérisque 198-200 (1991), 67-76.

[10] Y. BILU, Limit distribution of small points on algebraic tori, Duke Math. J. 89 (1997), $465-476$.

[11] F. Blanchard, $\beta$-expansions and Symbolic Dynamics, Theoret. Comput. Sci. 65 (1989), 131-141.

[12] D. Boyd, The Maximal Modulus of an Algebraic Integer, Math. Comp. 45 (1985), $243-249$

[13] D. Boyd, Salem numbers of degree four have periodic expansions, in Théorie des Nombres - Number Theory, Eds. J.M. de Koninck and C. Levesque, Walter de Gruyter \& Co., Berlin and New York (1989), 57-64.

[14] D. Boyd, On beta expansions for Pisot numbers, Math. Comp. 65 (1996), 841-860.

[15] D. Boyd, On the beta expansion for Salem numbers of degree 6, Math. Comp. 65 (1996), 861-875.

[16] D. Boyd, The beta expansions for Salem numbers, in Organic Mathematics, Canad. Math. Soc. Conf. Proc. 20 (1997), A.M.S., Providence, RI, 117-131.

[17] A. Brauer, On algebraic equations with all but one root in the interior of the unit circle, Math. Nachr. 4 (1950/51), 250-257.

[18] F. Carlson, Über Potenzreihen mit ganzzahlingen Koeffizienten, Math. Z. 9 (1921), $1-13$. 
[19] P. Dienes, The Taylor Series, Clarendon Press, Oxford (1931).

[20] P. Erdös and P. Turán, On the distribution of roots of polynomials, Ann. Math. 51 (1950), 105-119

[21] L. Flatto, J.C. Lagarias and B. Poonen, The zeta function of the beta transformation, Ergod. Th. and Dynam. Sys. 14 (1994), 237-266.

[22] Ch. Frougny, Confluent linear numeration systems, Theoret. Comput. Sci. 106(2) (1992), 183-219.

[23] Ch. Frougny, Number Representation and Finite Automata, London Math. Soc. Lecture Note Ser. 279 (2000), 207-228.

[24] Ch. Frougny, Numeration systems, chapter 7 in [31].

[25] W.H.J Fuchs, On the Zeros of Power Series with Hadamard Gaps, Nagoya Math. J. 29 (1967), 167-174.

[26] T. Ganelius, Sequences of analytic functions and their zeros, Arkiv för Math. 3 (1958), $1-50$.

[27] J.-P. Gazeau and J.-L. Verger-Gaugry, Geometric study of the beta-integers for a Perron number and mathematical quasicrystals, J. Théorie Nombres Bordeaux 16 (2004), 125-149.

[28] C.P. Hughes and A. Nikeghbali, The zeros of random polynomials cluster uniformly near the unit circle, arXiv:math/0406376v3 [math.CV] 3 Jun 2007.

[29] D.H. Lehmer, Factorization of certain cyclotomic functions, Ann. of Math. 34 (1933), $461-479$. 
[30] D. Lind, The entropies of topological Markov shifts and a related class of algebraic integers, Erg. Th. Dyn. Syst. 4 (1984), 283-300.

[31] M. Lothaire, Algebraic Combinatorics on Words, Cambridge University Press, (2003).

[32] K. MAHLER, Arithmetic properties of lacunary power series with integral coefficients, J. Austr. Math. Soc. 5 (1965), 56-64.

[33] M. MARden, The geometry of the zeros of a polynomial in a complex variable, Amer. Math. Soc. Math Surveys Number III (1949).

[34] M. Mignotte, Sur un théorème de M. Langevin, Acta Arithm. LIV (1989), 81-86.

[35] M. Mignotte, On the product of the Largest Roots of a Polynomial, J. Symbolic Computation 13 (1992), 605-611.

[36] W. PARry, On the $\beta$-expansions of real numbers, Acta Math. Acad. Sci. Hungar. 11 (1960), 401-416.

[37] G. Polya Sur les séries entières à coefficients entiers, Proc. London Math. Soc. 21 (1923), 22-38.

[38] PythéAs FogG, Substitutions in Dynamics, Arithmetics and Combinatorics, Lect. Notes Math. 1794, Springer (2003).

[39] A. RÉNyI, Representations for real numbers and their ergodic properties, Acta Math. Acad. Sci. Hungar. 8 (1957), 477-493.

[40] G. RHIN and Q. WU Integer transfinite diameter and computation of polynomials, preprint (2007).

[41] R. Salem, Power series with integral coefficients, Duke Math. J. 12 (1945), 153-172. 
[42] J. Schmeling, Symbolic dynamics for $\beta$-shift and self-normal numbers, Ergod. Th. Dynam. Sys. 17 (1997), 675-694.

[43] E.S. Selmer, On the irreducibility of certain trinomials, Math. Scand. 4 (1956), 287302

[44] C. Sмүтн, On the product of the conjugates outside the unit circle of an algebraic integer, Bull. London Math. Soc. 3 (1971), 169-175.

[45] B. SolomyaK, Conjugates of beta-numbers and the zero-free domain for a class of analytic functions, Proc. London Math. Soc. (3) 68, (1993), 477-498.

[46] G. SzegÖ, Über Potenzreihen mit endlich vielen verschiedenen Koeffizienten, Sitzungberichte Akad. Berlin (1922), 88-91.

[47] G. SzegÖ, Tschebyscheffsche Polynome und nichtvorsetzbare Potenzreihen, Math. Ann. 87 (1922), 90-111.

[48] Y. TAKAhashi, $\beta$-transformation and symbolic dynamics, Lect. Notes Math. 330 (1973), 455-464.

[49] J.-L. Verger-Gaugry, On gaps in Rényi $\beta$-expansions of unity for $\beta>1$ an algebraic number, Annales Institut Fourier 56 (2006), 2565-2579.

[50] M. Weiss and G. Weiss, On the Picard property of lacunary power series, Studia Math. 22 (1963), 221-245.

[51] O. Yамамото, On some bounds for zeros of norm-bounded polynomials, J. Symbolic Computation 18 (1994), 403-427. 
Author's address: Institut Fourier, CNRS UMR 5582, Université de Grenoble I, BP 74, 38402 Saint-Martin d'Hères, France.

email: jlverger@ujf-grenoble.fr 